\title{
Variations
}

Variations

Revue internationale de théorie critique

$9 / 10 \mid 2007$

Les frontières de la politique

\section{Un triste visage de la république : le musée du quai Branly}

Julien Bordier

\section{OpenEdition}

Journals

\section{Édition électronique}

URL : http://journals.openedition.org/variations/480

DOI : 10.4000/variations.480

ISSN : 1968-3960

Éditeur

Les amis de Variations

Édition imprimée

Date de publication : 1 juin 2007

Pagination : 119-134

Référence électronique

Julien Bordier, «Un triste visage de la république : le musée du quai Branly », Variations [En ligne], 9/10 | 2007, mis en ligne le 01 décembre 2012, consulté le 19 avril 2019. URL : http://

journals.openedition.org/variations/480 ; DOI : 10.4000/variations.480

Ce document a été généré automatiquement le 19 avril 2019

Les ami•e•s de Variations 


\title{
Un triste visage de la république : le musée du quai Branly
}

\author{
Julien Bordier
}

1 Outils centraux des politiques culturelles et porteurs d'une histoire lourde, les musées nationaux français connaissent aujourd'hui des mutations importantes alors que leur sort semble être abandonné au débat d'experts et à la gestion néo-managériale. On trouve pourtant dans ces institutions un terrain fertile à l'analyse sociologique. Serait-il passé de mode ? La sociologie française de l'analyse du musée, de la réception des objets exposés et du ou des publics semble avoir arrêté son élaboration intellectuelle sur L'Amour de l'art de Bourdieu et Darbel' ${ }^{1}$. Une sociologie dans laquelle le visiteur intégrerait pleinement les rapports de domination en jeu dans ses relations aux pratiques et expressions culturelles.

2 La sociologie de l'art et des musées s'est beaucoup intéressée aux comportements du ou des publics et les avant-gardes artistiques ont largement remis en question l'institution en elle-même, historiens et philosophes ${ }^{2}$ se sont penchés sur l'élaboration du musée et de sa participation à la construction des mémoires et des identités.

3 Ne faudrait-il pas tenter de rassembler ces différentes approches pour ouvrir une réflexion critique sur l'institution muséale et ses transformations? Cette institution ne serait-elle pas révélatrice des symptômes de la conjoncture politique actuelle?

4 Travailler les problématiques culturelles et artistiques3 permet la mise en évidence des rapports de domination symbolique, mais offre également une perspective enthousiaste. Le champ culturel, celui de la création, de la pratique et de l'interprétation, est également celui du renouvellement permanent et de la remise en question des schémas existants. S'il existe bien des inégalités culturelles, au niveau de l'accès à une certaine Culture, chacun et chacune dispose de ses propres pratiques culturelles pour échapper au monde ou le redéfinir. En ce sens, le musée public nous fournit un terrain où penser la tension politique. Le succès des musées, en termes de fréquentation, montre en tout cas que l'institution est loin d'être délaissée par ses visiteurs et utilisateurs.

5 Il importera donc ici de reposer la question du musée en la plaçant dans son contexte actuel, marqué par l'ouverture récente d'une nouvelle institution publique à Paris: le musée du quai Branly et l'espace polémique que ses orientations muséographiques 
peuvent ouvrir. Cette ouverture doit être mise en perspective avec la « modernisation des modes de gestion" des musées nationaux, en particulier leur transformation en Établissement Public Administratif (EPA) à l'autonomie gestionnaire renforcée, et la redéfinition du paysage muséal public français qui l'accompagne.

Nous questionnerons ce nouveau paysage, en le rattachant à une histoire politique des musées publics et en essayant de comprendre quels enjeux s'y manifestent. En particulier, la question de la relation de pouvoir sera ici abordée. Nous nous demanderons quelles nouvelles formes de dominations, ou enjeux de pouvoir, voient le jour sur ce terrain et quelles résistances inédites peuvent venir s'y opposer et ouvrir un espace public à la fois culturel et critique.

\section{Musée, république et démocratie}

7 L'histoire du musée public est celle d'un ensemble de contradictions. Elle est largement attachée, dans le contexte français, à la construction d'une idéologie républicaine et d'une identité nationale. Notons une contradiction originelle datant de la Révolution française. L'ouverture publique des collections royales précède l'avènement de la république. C'est d'abord un projet monarchique. Alors que les collections étaient jusqu'ici inaccessibles, l'idée d'une ouverture publique d'un Muséum Royal à Paris est envisagée par le comte d'Angiviller, surintendant du roi Louis $\mathrm{XVI}^{3}$. Ce projet est un signe fort adressé à une bourgeoisie contestataire : le pouvoir aristocratique décadent cherche à créer des espaces de liberté qui seraient autant de soupapes contre-révolutionnaires. La monarchie mise à bas, il suffira donc aux républicains de reprendre cette idée à leur compte, de stigmatiser le secret des collections royales et déclarer qu'elles sont désormais propriété du peuple et non du roi. Deux citations d'un fervent partisan de la naissance de cette forme de politique culturelle républicaine, l'Abbé Grégoire, sont à ce titre assez éloquentes : «Tous ces objets précieux qu'on tenait loin du peuple, ou qu'on ne lui montrait que pour le frapper d'étonnement ou de respect lui appartiennent $t^{4}$ », et aussi : «La France entière est persuadée que le dépérissement des arts serait celui de son existence et leur tombeau celui de la libertés ${ }^{\prime}$. À partir de ce projet, prend forme non sans tension ${ }^{6}$ une politique du patrimoine, sorte d'ébauche et d'ancêtre des politiques culturelles républicaines. On peut qualifier son ambition de démocratique dans la mesure où le maître mot de cette politique est l'ouverture au public, ou en d'autres termes, la création d'un espace public culturel. Reste à savoir comment cette dimension d'ouverture démocratique s'est perpétuée et sous quelle forme. Ainsi voit le jour un patrimoine républicain et un programme de conservation attachés et initiés par le nouveau régime. Son enjeu politique est la construction d'une identité nationale et républicaine, qui nous intéressera particulièrement ici.

Pour comprendre sa dimension politique, et donc tendue et conflictuelle ${ }^{7}$, la question de la conservation patrimoniale républicaine doit être posée à partir d'un moment fondateur qu'est le vandalisme révolutionnaire. Une grande partie des expressions artistiques aristocratiques à la portée du peuple sont d'ordre signalétique ou immobilier ${ }^{8}$. Elles sont à même la rue et porteuses de symboles aristocratiques. La chute de la monarchie s'accompagne d'un processus d'iconoclasme révolutionnaire où ces symboles de l'Ancien Régime sont attaqués et détruits. Mettre un terme à cette vague de destruction et imposer la politique patrimoniale a donc nécessité un certain volontarisme politique. Il serait trop long ici, et quelque peu éloigné de notre objet, d'engager une réflexion approfondie sur le vandalisme. Notons tout de même que celui-ci est sans visage et que le rapport de force 
qu'il exprime est diffus. Gardons en tête que la conservation patrimoniale républicaine trouve là un instant fondateur et se construit sur cet antagonisme entre destruction et conservation. De plus, cette question s'actualise dans les problématiques posées par l'existence du musée du quai Branly. Quelles questions sont soulevées par la mise en exposition de certains objets dont l'utilisation rituelle les vouait à la destruction? C'est la conservation qui remportera le combat idéologique entre la plèbe vandale et les partisans d'une république éclairée, liée au projet des Lumières, dans laquelle les Beaux-Arts se voient assigner une mission de civilisation. Il est d'ailleurs important de noter que la mise en place d'un programme de conservation et de protection des arts permet à la bourgeoisie révolutionnaire française de s'acheter un véritable "brevet de civilisation ${ }^{9}$ " aux yeux de ses voisins européens. Toutefois le musée ne fait pas l'unanimité au sein des amateurs et défenseurs des arts. Certains des plus réactionnaires, Quatremère de Quincy à leur tête, y voient une mise en danger des œuvres dans la mesure où elles se trouvent extériorisées de leur contexte.

C'est à cette période que le musée se voit assigner des missions précises et politiques. C'est une institution dévolue à l'ouverture publique, mettant en scène la propriété collective, incarnant un idéal esthétique, démontrant la sagesse de l'Etat. Il est organisé dans une optique pédagogique dans la mesure où il porte un projet éducatif, et mis en perspective avec un apprentissage de la liberté politique. On peut d'ailleurs voir cette première mouture du musée républicain comme en discussion avec le projet des Lumières. On y retrouve d'une part Kant et la Critique de la faculté de juger ${ }^{10}$ avec les idées de suspension de la finalité - ou transcendance - de l'œuvre, d'administration raisonnée et d'universalité esthétique. D'autre part, on peut voir à travers la dimension éducative et proprement politique du musée le projet, élaboré par Schiller dans les Lettres sur l'éducation esthétique de l'homme ${ }^{11}$, d'enseignement de la liberté par la mise en place d'un Etat esthétique, annonçant la République éclairée. D'un point de vue davantage organisationnel, le musée issu de la Révolution est marqué par le centralisme qui est alors de mise. Paris construit sa place de capitale des arts. Les dépôts, les prêts et les envois d'œuvres en province sont difficiles et très réglementés. Retenons donc de cette période la dimension démocratique (ouverture au public et propriété collective), la dimension éducative (enseignement d'un goût artistique) et la dimension centralisatrice (concentration du pouvoir politique et de la pré-administration culturelle à Paris).

10 Récupéré par les aristocrates restaurateurs et les deux Empires, réadapté par les républicains par la suite, le musée national trouve finalement sa place et sa légitimité. Il devient une institution incontournable. Le jeu des représentations sociales liées au musée évolue, se construit, s'enrichit et se complexifie. Peu à peu, le projet émancipateur des Lumières se cristallise. On peut reprendre les trois catégories mentionnées plus haut (éducation, centralisation, démocratisation) pour mettre en évidence cette cristallisation.

11 La dimension éducative est détournée de son public plébéien et orientée vers l'éducation artistique pour les apprentis artistes, en vue de créer une puissante école française et un goût national. L'éducation devient acculturation. La diversité des collections est abolie, instituant une frontière entre arts et sciences. La dimension centralisatrice, qui se voulait une garantie d'une répartition raisonnée des collections sur le territoire, se fige dans la formation d'un corps d'experts parisiens, lié à l'appareil d'Etat. Les Académies confisquent l'esthétique et deviennent des arènes de luttes de pouvoir. Cette contrition administrative et intellectuelle éclate au grand jour avec des conflits comme celui autour de l'Impressionnisme. Enfin, la dimension démocratique est elle-même restreinte. Alors 
que l'amateurisme artistique prend corps dans la bourgeoisie, un public élitaire cherche à chasser les vagabonds qui trouvent dans le musée un abri urbain ${ }^{12}$.

Ces trois dimensions se cristallisent particulièrement sous la IIIe République, qui ne remet pas en question la manière dont l'Empire lui-même s'était réapproprié les politiques publiques muséales. À cela s'ajoute le positivisme philosophique propre à ce régime, qui trouve dans le musée un lieu de prédilection pour présenter l'Histoire comme un mouvement continu et ordonné. Cela se manifeste particulièrement à travers la mainmise des Académies sur les arts à cette époque. Il y a un blocage artistique et politique directement lié aux enjeux de pouvoirs propres aux appareils d'État ${ }^{13}$. Remarquons enfin, comme le fait Habermas ${ }^{14}$, que la construction de l'espace public bourgeois est fortement liée à l'amateurisme et la critique d'art. Le musée national est devenu un visage de la République. Le musée du quai Branly en porte les traits, comme nous allons le voir : c'est un musée parisien, voulu par le président de la République, et qui se propose de nier l'histoire des objets qu'il expose. L'enjeu de ce rappel historique est de montrer en quoi le musée est une institution politique : il est au cœur de tensions entre la récupération bourgeoise et l'appropriation populaire, la conservation et le vandalisme, la fermeture et l'ouverture, la mise en question de l'objet et la réponse patrimoniale, la continuité positiviste et le bouleversement des schémas esthétiques. Nous tenterons de voir comment ces tensions prennent forme et s'actualisent au sein du musée du quai Branly et du projet dont il est porteur.

13 Nous parlons bien sûr ici d'un background culturel et historique qui fonde les représentations sociales liées au musée public et sa légitimité. L'administration culturelle a connu de vastes mutations, notamment sous les ministères Malraux et Lang ${ }^{15}$. Toutefois, si l'aide à la création a véritablement émergé grâce à ces ministres, leur politique muséale n'a pas été révolutionnaire, reprenant à leur compte une diffusion « démocratique » des œuvres envisagée dès l'émergence du muséum républicain de la Révolution. Notons tout de même l'élaboration et la mise en place d'un réseau institutionnel mutualiste et attaché au service public - mais centralisé - autour de la Direction des Musées de France et de la Réunion des Musées Nationaux. Les politiques culturelles de la Ve République sont moins audacieuses que celles du Front Populaire, qui permit la récupération des musées par la nébuleuse associative de l'époque ${ }^{16}$.

\section{Le musée du quai Branly, de la polémique au consensus}

14 L'ouverture du musée du quai Branly, au printemps 2006, a été un événement majeur de la vie culturelle française, et de la vie muséale en particulier. En France, un large consensus médiatique s'est constitué autour de son ouverture, alors même que sa préfiguration s'est réalisée dans la tension et le tiraillement. Le débat s'est centré sur l'opposition entre la contextualisation des objets et leur mise en valeur esthétique. On pourrait donc, bien que nous soyons conscients du simplisme de cette présentation, distinguer deux camps. Les anthropologues, d'une part, souhaitent faire apparaître la dimension culturelle d'un objet, ou se servir du tremplin proposé par un objet pour tenir un discours anthropologique. De l'autre côté, on trouve amateurs d'art, galeristes et autres amoureux des arts extra-européens, qui donnent la priorité à la dimension esthétique d'un objet, c'est-à-dire sa beauté plastique et l'émotion esthétique procurée 
par sa contemplation. Il s'est joué là un débat franco-français où éclataient les rivalités disciplinaires entre science et art.

C'est la deuxième option qui a été retenue au musée du quai Branly : celle de la mise en valeur de l'esthétisme. L'objet de ce texte ne sera pas de démontrer et déconstruire ce fait. D'autres s'y sont déjà employés ${ }^{17}$. Nous essaierons plutôt ici de proposer une lecture du paysage muséal français, à partir de l'exemple du musée du quai Branly et à la lumière de l'aperçu historique dressé plus haut. Nous nous demanderons donc dans quelle mesure ce musée s'inscrit dans le projet républicain français, ce que cela implique en termes de patrimonialisation et quelles brèches réflexives et critiques ouvre cette nouvelle institution. Comprendre l'impact et les enjeux liés au musée du quai Branly, c'est tenter une analyse en termes de pouvoir et d'anti-pouvoir. Cette institution est en effet révélatrice, ou symptomatique, des fonctions assignées aux musées nationaux en particulier et des politiques culturelles républicaines en général. On traitera ici quatre idées principales : la technocratie esthétique, le centralisme culturel, le rôle du dirigeant politique et la question de l'homogénéité sociale dans la République française.

Comme nous l'écrivions plus haut, au musée du quai Branly, c'est le point de vue de l'esthétisme qui l'a emporté sur le point de vue contextualisant. Cette orientation est directement inspirée de la volonté du collectionneur-marchand-amateur-expert Jacques Kerchache, aujourd'hui décédé, proche de Jacques Chirac. Kerchache, jouant de ses rapports privilégiés avec Chirac, a appuyé de toutes ses forces la création de ce musée et insisté pour que ce soient des « chefs-d'œuvre ${ }^{18}$ » qui y soient exposés. Ainsi, pendant la préfiguration du musée, les pré-équipes du musée du quai Branly se servaient dans les réserves du musée de l'Homme ou du Musée National des Arts d'Afrique et d'Océanie (MNAAO), pour constituer leurs propres collections.

Le dépouillement des réserves du MNAAO et du musée de l'Homme s'inscrit dans une volonté unilatérale de centralisation des collections. Alors que les deux institutions réussissaient tant bien que mal à jouer un rôle complémentaire: presque strictement ethnographique au musée de l'Homme et mise en perspective esthétique au MNAAO, le musée du quai Branly éteint la dimension anthropologique des objets qui sont exposés. Il ferme ainsi une possibilité d'ouverture sur l'altérité. Si la dimension plastique peut avoir un intérêt particulier pour participer à un dialogue esthétique entre les cultures, le visiteur est perdu dans les expressions d'un Autre qu'il n'a pas l'occasion de rencontrer. Par exemple, comment comprendre l'omniprésence des dents de cochon dans les sculptures du Vanuatu si le rôle social et politique de l'échange de cochons hermaphrodites aux canines recourbées dans la société de grade n'est nulle part explicité?

Le musée du quai Branly est également un cas représentatif de la création républicaine d'une nouvelle institution muséale. La Ve République voit chacune de ses présidences marquées par l'ouverture d'un grand musée public et parisien. L'ouverture d'un nouveau musée est évidemment un geste hautement politique. Mais n'est-il pas uniquement institutionnel quand c'est le président lui-même qui met son poids, sans et contre l'avis des personnes potentiellement concernées? La politique institutionnelle que nous connaissons et vivons n'est-elle pas l'expression d'une oligarchie, et non pas d'une démocratie, comme l'écrit Rancière ${ }^{19}$, c'est-à-dire une politique anti-politique ? Prenons le risque d'écrire ici que l'ouverture d'un musée sous la Ve République est un acte antidémocratique, une négation des tensions politiques qui devraient pourtant être au cœur d'une réflexion complète sur le fait de conserver et d'exposer des objets. L'ouverture d'un 
musée, c'est le « fait du prince » : Pompidou eut le musée d'art moderne, Giscard le musée d'Orsay, Mitterrand le grand Louvre et Chirac le quai Branly ${ }^{20}$.

Une quatrième dimension se trouve au croisement de ces trois premières : la question de l'homogénéité sociale dans la République. Esthétisation contre contextualisation, centralisation contre diffusion, réponse dirigeante contre question démocratique, sont autant d'antagonismes qui mettent en évidence la manière dont la République française se refuse de penser les diversités, le mouvement et les contradictions sociales et politiques. Nous sommes donc face à un projet radicalement franco-républicain, illustrant l'incapacité ou le refus des institutions à mettre en place des projets d'émancipations collectives et individuelles. Mais présenter ces quatre dimensions du pouvoir, c'est négliger les brèches qu'elles ouvrent malgré elles et les possibilités de dépassement que le musée est susceptible d'apporter. L'approche esthétique exacerbée du musée du quai Branly conduit inexorablement à une envie de savoir et de connaissance, il suffit de constater l'attrait du public pour les quelques dispositifs de médiation présents dans les salles du musée. L'émancipation intellectuelle se comprend dans l'égalité des intelligences: le manque de contextualisation au musée du quai Branly, s'il prend le risque de l'interprétation ethnocentriste et exotique, ouvre une voie critique pour la revendication de l'éducation populaire au sein du musée public. Appuyons-nous ici sur Rancière et ses concepts de "partage du sensible » et "d'égalité des intelligences ». Les biens culturels patrimonialisés, conservés, muséifiés participent à un partage du sensible. Celui-ci repose sur un commun partagé et des parts exclusives. "Ce système d'évidences sensibles qui donnent à voir en même temps l'existence d'un commun et les découpages qui y définissent les places et les parts respectives ${ }^{21}$. " Chacun ne peut avoir part au commun qu'en fonction de sa place dans la société. Quant à l'égalité des intelligences et l'émancipation intellectuelle ${ }^{22}$, elle nous permet de comprendre que l'espace public ouvert par le musée, potentiellement plébéien et critique, peut être occupé de façon polémique et donc politique par ses visiteurs. La soif de savoir des visiteurs n'est pas la seule brèche dans l'édifice du musée. Le centralisme culturel et politique exprimé par le musée du quai Branly appelle quant à lui une réaction politique. Le dépouillement des collections du MNAAO et du musée de l'Homme ne s'est d'ailleurs pas fait sans réaction des établissements concernés. La volonté dirigeante de création de cet établissement met en évidence une aristocratie d'Etat dont l'existence même entre en conflit avec les aspirations démocratiques et appelle donc une parole critique. Enfin, le recoupement de ces dimensions permet de mettre en évidence les fermetures politiques propres à la conception républicaine française.

Pour conclure sur ces possibilités de dépassements offertes par l'ouverture du musée du quai Branly, par le fonctionnement des musées en général et des représentations sociales qui leur sont attachées, citons Aminata Traoré, ancienne ministre de la Culture et du tourisme du Mali et « altermondialiste », à propos du musée du quai Branly :

Nos œuvres ont droit de cité là où nous sommes, dans l'ensemble, interdits de séjour. À l'intention de ceux qui voudraient voir le message politique derrière l'esthétique, le dialogue des cultures derrière la beauté des œuvres, je crains que l'on soit loin du compte. Un masque africain sur la place de la République n'est d'aucune utilité face à la honte et à l'humiliation subies par les Africains et les autres peuples pillés dans le cadre d'une certaine coopération au développement. Bienvenue donc au Musée de l'interpellation qui contribuera - je l'espère - à édifier les opinions publiques française, africaine et mondiale sur l'une des manières dont l'Europe continue de se servir et d'asservir d'autres peuples du monde tout en prétendant le contraire ${ }^{23}$. 


\section{Le musée du quai Branly et la question coloniale}

21 républicaine, et puisqu'il y est question d'expressions culturelles non-occidentales, provenant des «pays du Sud», il reste une question centrale: celle de la mémoire coloniale et de sa patrimonialisation. On attend d'un musée dont les collections ont un lien avec l'histoire coloniale qu'il propose une réflexion complète et accessible sur le rapport à l'Autre ${ }^{24}$, comme le propose par exemple le Tropenmuseum d'Amsterdam. On pourrait traiter cette question selon une première entrée «coloniale » et une seconde « postcoloniale ». La première se propose de revenir sur l'histoire coloniale et de mettre en évidence les procédés permettant de la masquer, la seconde s'interroge sur les formes de néocolonialisme à l'œuvre au musée du quai Branly. On doit d'abord noter que la majorité des collections publiques françaises est issue de collectes réalisées dans le cadre de la colonisation. On pourrait donc s'attendre à une réflexion sur la provenance des objets. Elle est à peine suggérée. Plus dérangeant encore, elle semble même digérée par la muséographie. L'espace muséal est divisé en zones de couleurs au sol, divisant arbitrairement des régions du globe, telle une carte coloniale aux frontières tracées à la règle et au compas : un découpage propre à l'œil du dominant ${ }^{25}$. Il y aurait pourtant tout un discours à construire et déconstruire autour de l'origine des collections et de la question du pillage ${ }^{26}$. Question d'autant plus importante que le pillage perdure et que le musée du quai Branly, en sanctuarisant ces objets et en devenant un acheteur potentiel sur ce marché, l'accentue et devient partie prenante dans les jeux spéculatifs.

Le musée pourrait ou devrait être un lieu de réflexion sur l'Autre et sur la question des identités. L'exposition temporaire D'un regard l'autre ${ }^{27}$ laisse le visiteur sur sa faim malgré un sujet attirant. Le regard du colonisé y est tout simplement occulté. Seul le point de vue occidental est proposé, celui des explorateurs, celui de l'exotisme porteur de mythes: mythe du bon sauvage dans les représentations idéalisées de l'ailleurs et de l'autre; mythe du mauvais sauvage dans la "salle des armes", menaçante et plongée dans l'obscurité. Cette exposition inaugurale doit être interprétée comme un manifeste du musée du quai Branly: négation de l'histoire coloniale, mise en scène du sauvage et glorification du regard de l'artiste occidental considéré comme le regard juste. Les objets présentés en fin de parcours sont sélectionnés en fonction de leur "pedigree ", c'est-àdire en fonction du prestige de l'artiste occidental qui en a été le propriétaire.

Dans les salles d'expositions permanentes, si le point de vue esthétisant peut être intéressant, il est très étonnant que la dimension culturelle des objets exposés soit quasiment tue et implicitement déformée. La disposition des cartels - discrets, mal placés, laconiques - montre qu'il y a là une volonté claire de gommer une dimension anthropologique: un refus de penser les hommes et leurs histoires, dont l'histoire coloniale, derrière et avec les objets... un processus de fétichisation de l'objet culturel, une séparation du fait et du faire, de l'objet culturel produit et de son producteur.

Énonçons enfin que le moment colonial peut être considéré comme un moment fondateur dans la construction de la République française, un visage de la République. Le musée, institution républicaine, se doit donc - ou doit pouvoir avouer son incapacité à le faire de proposer une réflexion claire sur cette question. On pourrait en tout cas s'y attendre. Mais là encore, c'est la réification patrimoniale qui impose ses grilles de lectures, celles de 
la mémoire des vainqueurs, comme l'écrit Walter Benjamin. C'est là que s'expose la dimension postcoloniale, ou néo-coloniale, de ce musée.

On pourrait d'abord penser que la quasi-négation de la question coloniale n'est qu'une orientation définie par une droite décomplexée. Celle-là même qui réalisait ses essais nucléaires dans ses territoires outre-mer (1996), qui faisait voter une loi sur le rôle positif de la colonisation (2003), qui déterrait les lois coloniales quand les quartiers populaires s'insurgeaient (2005), qui proposait une gestion drastique et répressive des flux d'immigration provenant de ses anciennes colonies (2006). Pourtant, la préfiguration du musée du quai Branly s'est construite pendant une période de cohabitation, où la gauche gouvernementale aurait pu essayer d'orienter les choix scientifiques et muséographiques de l'institution, empêcher le démantèlement d'autres établissements. Mais un nouveau musée parisien se doit d'être la chasse gardée d'un grand président, pour le malheur des objets et documents qui y sont exposés et stockés. On constate ici une absence de clivage que l'on pourrait justement qualifier de « républicaine », parce qu'homogène.

Certains ministres du gouvernement Jospin se sont d'ailleurs illustrés brillamment. Catherine Trautman, alors homologue française d'Aminata Traoré, demandait à cette dernière d'autoriser l'achat d'une statuette Tial pour les collections du futur musée. «De peur de participer au blanchiment d'une ceuvre d'art qui serait sortie frauduleusement de notre pays ", Mme Traoré lui a proposé que la France achète la pièce, la restitue au Mali avant que celui-ci puisse la prêter temporairement au musée du quai Branly. L'ex-ministre malienne raconte :

Je me suis entendu dire, au niveau du Comité d'orientation, dont j'étais l'un des membres, que l'argent du contribuable français ne pouvait pas être utilisé dans l'acquisition d'une pièce qui reviendrait au Mali. Exclue à partir de ce moment de la négociation, j'ai appris par la suite que l'Etat malien, qui n'a pas de comptes à rendre à ses contribuables, a acheté la pièce en question en vue de la prêter au Musée ${ }^{28}$.

Une autre affaire du même type a agité les collections du musée du quai Branly, celle des sculptures $\mathrm{Nok}^{29}$. En 2000, des pièces statuaires provenant du Nigeria sont acquises par le musée pour enrichir ses collections. Mais l'exportation de ces objets est prohibée par la loi nigériane et inscrite sur la liste rouge de l'International Council of Museums (ICOM). Si le gouvernement français et le musée du quai Branly se cachent derrière la mauvaise foi des autorités nigérianes, le problème déontologique reste entier car les pièces sont toujours exposées. On peut donc redouter que, loin de songer à une quelconque répartition ou restitution des richesses culturelles non européennes, le musée du quai Branly continuera dans la voie de la dépossession symbolique et matérielle des objets susceptibles d'être exposés en ses murs.

Le musée du quai Branly, d'une certaine façon, c'est aussi la reconnaissance de la diversité culturelle, de ce qu'elle apporte au monde d'aujourd'hui et de ce en quoi elle est nécessaire, ne serait-ce que pour le respect de l'homme et pour la paix déclarait Jacques Chirac lors de l'inauguration du musée.

En place de diversité, le musée du quai Branly se propose de ne pas aborder la question coloniale à partir des objets qu'il expose. Ces objets n'opérant pas dans leur propre cadre culturel, leur mise en exposition soulève des situations inédites au niveau de l'institution muséale. Nous sommes donc à un carrefour : alors même que la république coloniale cherchait à légitimer ses actions par les bienfaits supposés de l'universalisme et la civilisation, universalisme et civilisation sont également des fondements idéologiques et de légitimation sociale du musée national et de la conservation patrimoniale. L'institution 
en elle-même peut-elle réussir à tenir un discours sur ses propres fondements ? Comment la République française pourrait-elle gérer son histoire coloniale? Comment un musée peut-il rendre compte d'une barbarie ? Ces raisonnements peuvent nous renvoyer à la pensée de Benjamin. On y trouve un attachement à une mémoire des vaincus, tel qu'il l'entend dans les thèses Sur le concept d'histoire: "Car il n'est pas de témoignage de culture qui ne soit en même temps un témoignage de barbarie. Cette barbarie inhérente aux biens culturels affecte également le processus par lequel ils ont été transmis de main en main ${ }^{30}$." Si l'institution se retrouve dans l'incapacité de créer un discours sur ces enjeux, les visiteurs, eux, peuvent le faire. Mais voyons d'abord rapidement, en plus des problématiques qui viennent d'être développées, le cadre économique et gestionnaire dans lequel s'inscrit ce nouveau musée.

\section{Le quai Branly dans le nouveau paysage muséal}

Le projet scientifique porté par le musée du quai Branly est largement polémique. De nombreux chercheurs en anthropologie contestent les orientations de l'établissement. Bernard Dupaigne, directeur du laboratoire d'Ethnologie du musée de l'Homme de 1991 à 1998 l'explique bien dans son enquête Le scandale des arts premiers ${ }^{31}$ : la main mise énarque sur la préfiguration et la gestion du musée, les lacunes des orientations scientifiques, les promesses non tenues en termes de recherche, les risques pris pour les objets et les documents transférés ${ }^{32}$, les conditions de « réquisition » et de transfert, les entraves à la recherche causées par le démantèlement des bibliothèques du musée de l'Homme et du MNAAO, le départ des cautions scientifiques des équipes de préfiguration, etc.

Le musée du quai Branly s'inscrit dans une vaste mutation du paysage muséal public français : une introduction du nouvel esprit du capitalisme dans la gestion des musées ${ }^{33}$. Celle-ci s'articule à la réforme de l'Etat et la remise en cause systématique des entreprises de service public. Les musées publics, et nationaux en particulier, espaces et lieux de travail dans lesquels il existait une relative souplesse administrative liée à une culture du service public culturel héritée du Front Populaire ${ }^{34}$ et des ministères Malraux ${ }^{35}$ et Lang, sont aujourd'hui démantelés, soumis aux objectifs de rentabilité, aux techniques de néomanagement et à une gestion du personnel stricte ${ }^{36}$. Le musée des ATP est fermé, le dialogue entre le musée de l'Homme (ministère de l'Éducation Nationale) et le MNAAO (ministère de la Culture) passé à la trappe. Un musée des cultures européennes et méditerranéennes bien distinct du quai Branly va voir le jour à Marseille. Cette distinction montre quelles frontières sont établies, et nuit au dialogue entre les cultures. Quant au bâtiment colonial du MNAAO, il deviendra une "Cité de l'immigration ».

D'un point de vue institutionnel, les musées nationaux sont peu à peu autonomisés financièrement et culturellement par leur transformation en EPA - et détachés des réseaux institutionnels de mutualisation des ressources et des besoins des différents musées. Le recours au mécénat privé s'en trouve encouragé, les missions de service public culturel sont remises en cause, profits et commercialisation deviennent des objectifs affichés, les processus sociaux d'appropriation publique sont perturbés, l'espace démocratique potentiel ouvert par le musée est restreint. 


\section{Pour un espace muséal public et critique}

32 Le musée est un espace politique. Il concentre une certaine esthétique, une certaine mémoire reconnues par l'État et se légitime par son ouverture démocratique. Une certaine tension se manifeste donc en son sein. Toutefois, son ouverture publique est contrariée. De manière historique, le musée est un espace contradictoire. Il est un outil qui répond au vandalisme, c'est donc un espace public contraint, une appropriation publique soumise à l'acceptation de certaines règles strictes. Elle le sera davantage par la mainmise idéologique des Académies sur les arts pendant la IIIe République. Elle le sera par l'administration culturelle centralisatrice sous la Cinquième. Mais l'ouverture publique a toujours été revendiquée, la légitimité du musée n'existe qu'à travers elle, et elle anime une tension en son sein.

En France, un étrange consensus semble entourer le musée du quai Branly, alors même que sa préfiguration faisait vivre un espace critique essentiel et que des voix s'élèvent de par le monde pour condamner ses orientations ethnocentristes. Dans cet espace, on aurait peut-être pu faire émerger la question coloniale. On pouvait naïvement espérer que le nouveau musée prendrait la question à bras le corps. Le quai Branly fait table rase du passé colonial, jetant délibérément aux oubliettes une mémoire qui nous permettrait de nous reconstruire d'un point de vue critique par rapport cette question. On s'attendait à des muséographies nouvelles dans lesquelles les pistes d'une réflexion seraient ouvertes. Au lieu de cela, le visiteur se trouve à nouveau invité dans un sanctuaire célébrant la République française et son président, dont les orientations muséologiques et gestionnaires semblent étouffer toute possibilité de dépassement.

Mais « les publics » n'ont sûrement pas dit leur dernier mot, et les politiques de gestion des publics mises en place par les musées sont loin de saisir les attentes intellectuelles et émancipatrices de ceux et celles qui franchissent le seuil des musées, entre amis, entre amants, en famille, au hasard, occasionnellement ou régulièrement ${ }^{37}$. La remise en cause de l'ouverture démocratique des musées n'est pas acceptée, comme l'a montré la mobilisation des artistes et des enseignants quand le musée du Louvre voulut remettre en question la gratuité du droit d'entrée qui leur est accordée. Aujourd'hui, le Louvre s'apprête à ouvrir une antenne à Abu Dhabi, dans les Émirats Arabes Unis, au milieu d'un complexe touristique, en échange de quelques valises de pétrodollars. Universalisme républicain ou diplomatie? "Nous ne servons pas les intérêts diplomatiques de la France, nous servons la France» déclare Serge Lemoine, président de l'EPA musée d'Orsay, pour apporter sa caution au projet ${ }^{38}$. Comme une réponse, les conservateurs se mobilisent et signent une pétition faisant suite à un article de Françoise Cachin intitulé Les musées ne sont pas à vendre ${ }^{39}$. Il paraît clair que l'introduction des objectifs de rentabilité financière dans la gestion des musées publics crée un terrain polémique inédit. Les résistances ici citées en sont la preuve, tout comme celles qui ont agité le musée de l'Homme pendant la préfiguration du musée du quai Branly. Elles restent toutefois des résistances assez corporatistes et au fond peu ouvertes sur le public. En somme, elles s'inscrivent ellesmêmes dans une idéologie républicaine typiquement française. Reste donc à savoir maintenant comment le public tentera de se réapproprier des institutions dont on l'a dépossédé symboliquement, et dont la raison d'être repose sur une dépossession mais aussi sur une envie d'appropriation collective : une appropriation qui se manifeste par la destruction, par la contemplation, par l'envie de comprendre, par le besoin de savoir. 
Reste à savoir comment le public du musée du quai Branly fera résonner et raisonner en ses murs les paroles d'Aimé Césaire, de Frantz Fanon, de Thomas Sankara, d'Amilcar Cabral, des résistances des peuples d'Afrique, d'Asie, d'Amérique et d'Océanie. Reste à imaginer comment l'égalité des intelligences et l'envie de l'émancipation trouveront les moyens de faire résonner partout où elles se manifestent - c'est-à-dire partout - la mémoire des vaincus.

\section{NOTES}

1. Minuit, 1966.

2. On pense ici par exemple à Jeanne Laurent, Pierre Nora, Dominique Poulot, Jean-Louis Déotte, Françoise Choay, Jean-Michel Leniaud, Bernard Deloche, Henri-Pierre Jeudy, Pascal Ory.

3. Dominique Poulot, 1997. Musée, nation, patrimoine, 1789-1815. Gallimard, p. 91.

4. Ibid., p. 131.

5. Bernard Deloche et Jean-Michel Leniaud, 1989. La Culture des sans-culottes. Éditions de Paris/ Presses du Languedoc, p. 8.

6. Babeuf écrit par exemple le 28 frimaire an II : «Les sans-culottes devraient bien couvrir de boue un certain rapport de l'abbé Grégoire. » (Poulot, 1997, p. 181).

7. On s'appuie ici sur Jacques Rancière, 2004 (1998). Aux Bords du politique, Folio Gallimard, 261p.

8. Dominique Poulot, 1997, p. 158.

9. Dominique Poulot, 1997, p. 176

10. Kant, 1995 (1790), Critique de la faculté de juger, Paris, Aubier.

11. Schiller, 1992 (1794), Lettres sur l'éducation esthétique de l'homme, Paris, Aubier.

12. François Mairesse, 2005. Le droit d'entrée au musée. Labor.

13. Jeanne Laurent, 1984. Arts et pouvoirs en France de 1793 à 1981, histoire d'une démission artistique. CIERC. Pierre Vaisse, 1995. La Troisième République et les peintres, Flammarion.

14. Jürgen Habermas, 1978. L'espace public. Payot.

15. Pour en savoir plus : Philippe Poirier, 2000. L'Etat et la culture en France au XXe siècle. Librairie générale française.

16. Pascal Ory, 1994. La Belle Illusion. Culture et politique sous le signe du Front Populaire, Plon.

17. Bernard Dupaigne, 2006. Le Scandale des arts premiers. La véritable histoire du musée du quai Branly. Mille et une nuits.

18. Il est pourtant essentiel de poser la question de la pertinence de cette terminologie, quand on évoque des objets provenant de cultures non-occidentales, où, dans certains cas, les termes d'art, d'œuvre d'art ou de chef-d'œuvre n'existent même pas.

19. Jacques Rancière, 2005.

20. André-Hubert Mesnard, L'Évolution de la politique culturelle sous la Ve République et la qualification de service public culturel, in Robert-Edouard Charlier, 1981, Service public et libertés, pp. 475-494.

21. Jacques Rancière, 2000. Le partage du sensible. La Fabrique, p. 12.

22. Jacques Rancière, 2004 (1987). Le maître ignorant. 10/18, 234p. et 2005, La haine de la démocratie. La Fabrique, 106p. 
23. Aminata Traoré, 30 juin 2006, « CEuvres de l'esprit n'entendez-vous pas les cris des noyés de l'émigration ", L'Humanité. Sur les rapports Nord-Sud, Aminata Traoré a également écrit Le viol de l'imaginaire (2002, Fayard) et L'Étau (1999, Actes Sud).

24. Pour approfondir cette proposition, nous proposons les lectures suivantes: Frantz Fanon, 1971 (1952). Peau noire, masques blancs. Éditions du Seuil ; Sally Price, 1995. Arts primitifs, regards civilisés. Ensb-a ; Edward W. Saïd, 2000. Culture et impérialisme, Fayard/Le monde diplomatique, et Roger Somé, 1998. Art africain et esthétique occidentale, L'Harmattan.

25. Peter Ferry, 2006. Le musée du Quai Branly, construction d'un lieu consacré à l'Autre. Mémoire de master en géographie, Université Paris XII.

26. Le trafic illégal d'œuvre d'art est reconnu comme étant la deuxième grande source de criminalité internationale après le trafic de drogue. Lire : Philippe Baqué, 1999. Un nouvel or noir. Paris Méditerranée ; Raymond Corbey, 2000. Tribal art trafic, a chronicle of taste and desire in colonial and postcolonial times, Royal tropical institute ; ICOM. 1994, Cent objets disparus, pillage en Afrique ; Emmanuel de Roux et Roland Pierre Paringaux, 1999. Razzia sur l'art. Vols, pillages, recels à travers le monde. Fayard.

27. Musée du quai Branly, 19/09/06 - 21/01/07.

28. Aminata Traoré, 2006, op. cit.

29. Emmanuel De Roux, «La route des sculptures Nok va-t-elle être coupée ?», 26 novembre 2000, in Lemonde.fr. Voir aussi : http://www.memoiredafrique.com/fr/nok/polemique.php

30. Walter Benjamin, 2000 (1972). «Sur le concept d'histoire » in E Euvres III, Folio Gallimard, p. 433.

31. Bernard Dupaigne, 2006. op. cit.

32. On appréciera le récit, mais on s'inquiétera de l'inondation des caves du musée du quai Branly, puisqu'il est zone inondable, dans lesquelles se trouvaient des archives qui venaient d'être sorties du musée de l'Homme.

33. Julien Bordier, 2004. Le service public culturel face à la "modernisation des modes de gestion" des Musées Nationaux, Mémoire de DEA en sciences de l'art, Université Paris I.

34. Remarquons que le musée de l'Homme et le musée des Arts et Traditions Populaires, aujourd'hui démantelé pour le premier et fermé pour le second, ont été ouverts sous le Front Populaire dans une dynamique d'éducation populaire.

35. Le Musée National des Arts d'Afrique et d'Océanie, aujourd'hui fermé, avait été ouvert par André Malraux.

36. Le recours systématique aux contrats précaires comme ajustement, et l'externalisation de certaines activités deviennent la règle dans la gestion du personnel des musées.

37. Sur les possibilités ouvertes par le musée, voir Peter Weiss, 1989. L'esthétique de la résistance. Klincksieck.

38. Interview sur France Inter le 9 janvier 2007.

39. Françoise Cachin, Jean Clair et Roland Recht, "Les musées ne sont pas à vendre ", 13 décembre 2006, et AFP, « Pétition contre les dérives commerciales du Louvre », 7 janvier 2007 in Lemonde.fr. 


\section{AUTEUR}

\section{JULIEN BORDIER}

Doctorant en sociologie 